\title{
Is Cholesteryl Ester Transfer Protein (CETP) Deficiency Atherogenic in Familial Hypercholesterolemia
}

Atherosclerosis is considered to be caused by many risk factors. Epidemiological studies have shown that the co-existence of multiple risk factors contributes to the progress in the disease more heavily than a single risk factor. A high level of LDL is a very strong risk factor for coronary heart disease (CHD), showing the high incidence of angina or myocardial infarction in familial hypercholesterolemia (FH). On the other hand, HDL is a negative risk factor for CHD. It was reported in epidemiological studies that a higher level of HDL correlates with a lower incidence of CHD. The mechanism for such an effect has been reported by several laboratories; the reverse cholesterol transport is enhanced by the addition of HDL, dose dependently in vitro. This result means that the accumulated cholesterol can be transported to plasma by HDL, which causes a decreased cholesterol content in cells and thus prevents foam cell formation. Therefore, it has been accepted that a higher level of HDL is preferable for the prevention of CHD. Concerning the different functions of the two lipoproteins, LDL and $\mathrm{HDL}$, it is very interesting to evaluate, the risk for CHD in patients presenting both high LDL and high HDL. These different functions from the aspect of atherogenecity or antiatherogenecity, were clinically studied in the patients with FH combined with cholesteryl ester transfer protein (CETP) deficiency. In this issue, Kamigaki et al reported a case of FH with CETP deficiency who did not show CHD (1).

See also p. 523.

The focus of their study was to evaluate the incidence of $\mathrm{CHD}$ and the grade of atherosclerosis in such patients. Haraki et al (2) have reported such double heterozygote cases, in which the effect of an increased HDL-C level caused by partial deficiency of CETP was insufficient to prevent CHD in FH. There are many studies on the atherogenecity of LDL both in basic and clinical research fields. A recent study on the Japanese-American male population demonstrated that a high level of HDL in heterozygous CETP deficiency was a positive risk factor (3). But the patients with a higher level of HDL showed the lower prevalence of CHD irrespective of CETP genotype. The results of CETP and CHD are conflicting. These data may suggest that both the quantity and quality of HDL particles determine antiatherogenic value. The reverse transport activity of HDL is reported to depend on the level, or the quality of HDL. A very high level of HDL-C of usually over $100 \mathrm{mg} / \mathrm{dl}$, can be observed, in homozygous CETP deficiency. In this case, HDL is different in size, composition, electrophoretical mobility and reverse transport activity. The HDL fraction is typically divided into two different subclasses, $\mathrm{HDL}_{2}$ and $\mathrm{HDL}_{3}$. $\mathrm{HDL}_{3}$ fraction consists of small particles with a low cholesterol content. $\mathrm{HDL}_{3}$ maturates to form $\mathrm{HDL}_{2}$ by trapping cellular cholesterol. Furthermore, the cholesterol ester in HDL is transported to the LDL or VLDL fraction by CETP in the plasma. The increased HDL observed in CETP deficiency is similar to $\mathrm{HDL}_{2}$ in size and composition. Probucol decreases the HDL-C level in plasma through an increase in CETP activity, however the HDL fraction still possesses a high level of the reverse transport activity. Therefore we cannot determine the function of the HDL level only by its quantity. Probucol is known to activate CETP and to reduce the plasma HDL content. On the other hand, probucol can dramatically reduce tendon xanthoma. These data indicate that the quality of HDL either, functional active or inactive, is very important. Furthermore, clinical studies, such as the Japan-Honolulu study (3) or the Omagari study (4) have shown that high level of HDL with heterozygous CETP deficiency is not antiatherogenic.

In FH with a high level of HDL, the $\mathrm{CHD}$ incidence is lower than with a low level of HDL (5). Therefore, the function of HDL is different between CETP deficiency and the other disease causing high level of HDL. These data suggest that treatment to increase the plasma HDL level should be applied on selected cases. It is very important to increase the amount of HDL possessing a high level of reverse transport activity. Furthermore, HDL has other functions, such as ameliorating endothelial dysfunction, attenuating lipid peroxidation and preventing coagulation. The correlation between these functions of HDL and the quality of HDL should be examined. The evaluation of not only quantity but also the quality of HDL is very important; in the future we should observe the quality carefully and understand its significance.

\section{Yasushi SAITO, MD The Second Department of Internal Medicine, Chiba University School of Medicine, 1-8-1 Inohana, Chuo-ku, Chiba 260-0856}

\section{References}

1) Kamigaki M, Tsuji M, Ishii J, et al. Familial hypercholesterolemia with cholesteryl ester transfer protein deficiency. Intern Med 37: 523, 1998.

2) Haraki T, Inazu A, Yagi K, Kajinami K, Koizumi J, Mabuchi H. Clinical characteristics of double heterozygotes with familial hypercholesterolemia and cholesteryl ester transfer protein deficiency. Atherosclerosis 132: 229, 1997. 
3) Zhong S, Sharp DS, Grove JS, et al. Increased coronary heart disease in Japanese-American men with mutation in the cholesteryl ester transfer protein gene despite increased HDL levels. J Clin Invest 97: 2917, 1996.

4) Hirano K, Yamashita S, Nakajima N, et al. Genetic cholesteryl ester transfer protein deficiency is extremely frequent in the Omagari area of
Japan. Marked hyperalphalipoproteinemia caused by CETP gene mutation is not associated with longevity. Arterioscler Thromb Vasc Biol 17: 1053, 1997.

5) Ferrieres J, Lambert J, Lussier-Cacan S, Davignon J. Coronary artery disease in heterozygous familial hypercholesterolemia patients with the same LDL receptor gene mutation. Circulation 92: 290, 1995. 